\title{
Characterisation of the static offset in the travelling wave in the cochlear basal turn
}

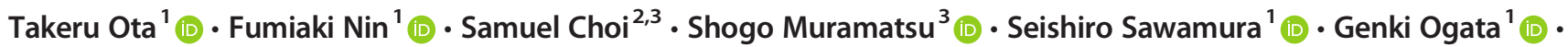 \\ Mitsuo P. Sato ${ }^{4}$ Katsumi Doi ${ }^{4} \cdot$ Kentaro Doi $^{5} \cdot$ Tetsuro Tsuji $^{5,6}$ (D) $\cdot$ Satoyuki Kawano ${ }^{2,5} \cdot$ Tobias Reichenbach $^{7}$ (D) • \\ Hiroshi Hibino ${ }^{1,2}$ (D)
}

Received: 3 February 2020 /Revised: 18 March 2020 / Accepted: 23 March 2020 / Published online: 22 April 2020

(C) The Author(s) 2020

\begin{abstract}
In mammals, audition is triggered by travelling waves that are evoked by acoustic stimuli in the cochlear partition, a structure containing sensory hair cells and a basilar membrane. When the cochlea is stimulated by a pure tone of low frequency, a static offset occurs in the vibration in the apical turn. In the high-frequency region at the cochlear base, multi-tone stimuli induce a quadratic distortion product in the vibrations that suggests the presence of an offset. However, vibrations below $100 \mathrm{~Hz}$, including a static offset, have not been directly measured there. We therefore constructed an interferometer for detecting motion at low frequencies including $0 \mathrm{~Hz}$. We applied the interferometer to record vibrations from the cochlear base of guinea pigs in response to pure tones. When the animals were exposed to sound at an intensity of $70 \mathrm{~dB}$ or higher, we recorded a static offset of the sinusoidally vibrating cochlear partition by more than $1 \mathrm{~nm}$ towards the scala vestibuli. The offset's magnitude grew monotonically as the stimuli intensified. When stimulus frequency was varied, the response peaked around the best frequency, the frequency that maximised the vibration amplitude at threshold sound pressure. These characteristics are consistent with those found in the low-frequency region and are therefore likely common across the cochlea. The offset diminished markedly when the somatic motility of mechanosensitive outer hair cells, the force-generating machinery that amplifies the sinusoidal vibrations, was pharmacologically blocked. Therefore, the partition offset appears to be linked to the electromotile contraction of outer hair cells.
\end{abstract}

Keywords Offset $\cdot$ Outer hair cells $\cdot$ Somatic motility $\cdot$ Travelling wave $\cdot$ Vibration

Takeru Ota and Fumiaki Nin contributed equally to this work.

Electronic supplementary material The online version of this article (https://doi.org/10.1007/s00424-020-02373-6) contains supplementary material, which is available to authorized users.

Fumiaki Nin

nin@med.niigata-u.ac.jp

Hiroshi Hibino

hibinoh@med.niigata-u.ac.jp

1 Department of Molecular Physiology, Niigata University School of Medicine, 1-757 Asahimachi-dori, Chuo-ku, Niigata 951-8510, Japan

2 AMED-CREST, AMED, Niigata 951-8510, Japan

3 Department of Electrical and Electronics Engineering, Niigata University, Niigata 950-2181, Japan
4 Department of Otolaryngology, Kindai University Faculty of Medicine, Osaka 589-8511, Japan

5 Department of Mechanical Science and Bioengineering, Graduate School of Engineering Science, Osaka University, Osaka 560-8531, Japan

6 Present address: Department of Advanced Mathematical Sciences, Graduate School of Informatics, Kyoto University, Kyoto 606-8501, Japan

7 Department of Bioengineering, Imperial College London, London SW7 2AZ, UK 


\section{Introduction}

In the mammalian hearing process, mechanical stimuli originating from a sound propagate through the middle ear and cause oscillatory pressure changes in the cochlea. The vibration elicits a wave that travels apically along the cochlear partition, a structure comprising the basilar membrane and overlying sensory hair cells and supporting cells $[15,50]$ (Fig. 1a). For a high-frequency stimulus, this travelling wave peaks near the cochlear base, whereas the wave reaches more apical positions at lower frequencies [50]. There are two types of hair cell, inner and outer; their cell bodies are bathed in perilymph, whereas their apical surfaces, from which the mechanosensitive hair bundles project, are exposed to a $\mathrm{K}^{+}$rich extracellular solution termed endolymph. The hair bundle vibration that results from motion of the cochlear partition deflects hair bundles, opens mechanoelectrical transduction channels to permit $\mathrm{K}^{+}$entry, and thus depolarises the hair cells $[20,37,40]$. Aside from triggering the release of a neurotransmitter by inner hair cells, the electrical excitation elicited by displacement of the hair bundles changes the length of cell bodies of outer hair cells. This phenomenon is called somatic motility or electromotility; it depends upon the motor protein prestin $[14,52]$ and can mechanically amplify cochlear partition oscillations [31,33].

A weak pure tone leads to a vibration of the cochlear partition that is greatly amplified near the site of the maximal vibration. Louder tones are progressively less amplified, resulting in a nonlinear compression of the vibration magnitude with respect to sound intensity [21, 43]. The nonlinear compression can be measured through laser interferometry and permits the detection of a million-fold range of sound pressure in normal hearing [33, 45]. For guinea pigs, cats, and chinchillas stimulated at 40 to $90 \mathrm{~dB}$ SPL, the response is related to the level of stimulation through a power law with a logarithmic slope of approximately one-third [43]. This property is likely to mirror the observed third-order change in open probability of the mechanoelectrical transduction a

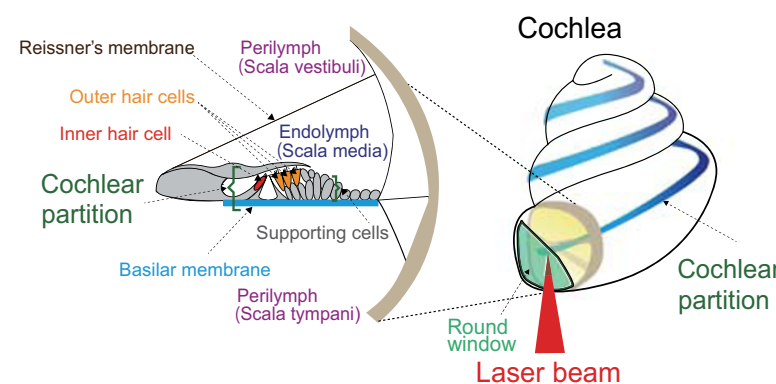

b

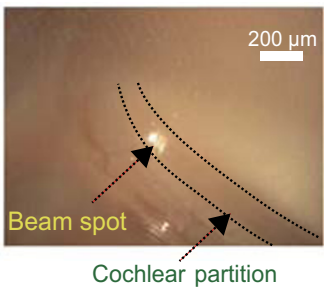

d

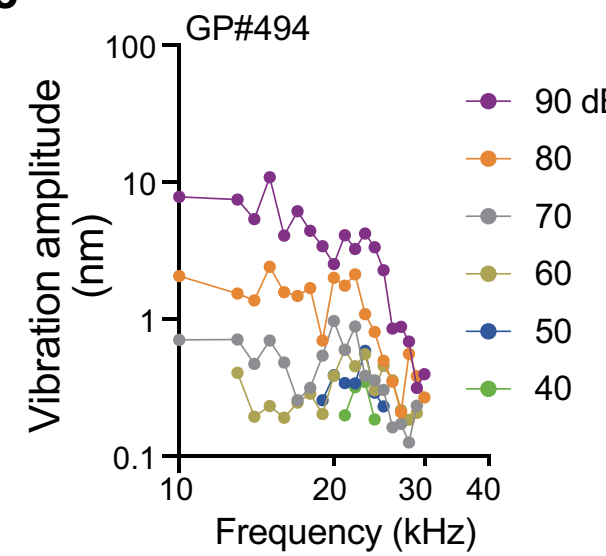

Fig. 1 Preparation of the cochlea and evaluation of the procedure. a Structure of the cochlea (right panel) and its cross-section (left panel). A laser beam directly irradiated a spot of the cochlear partition in the basal turn through the round window membrane. b The position of interferometric measurement. This experimental view of the cochlear basal turn in a guinea pig was obtained under a microscope equipped with our SPM vibrometer. As shown in this panel, the cochlear partition was detected as a shadowed belt (highlighted by two dotted lines), and the beam was focused around the centre of this landmark. c, $\mathbf{d}$ Tuning curves. Guinea pig 494 (GP\#494) was exposed to stimuli featuring a pair of

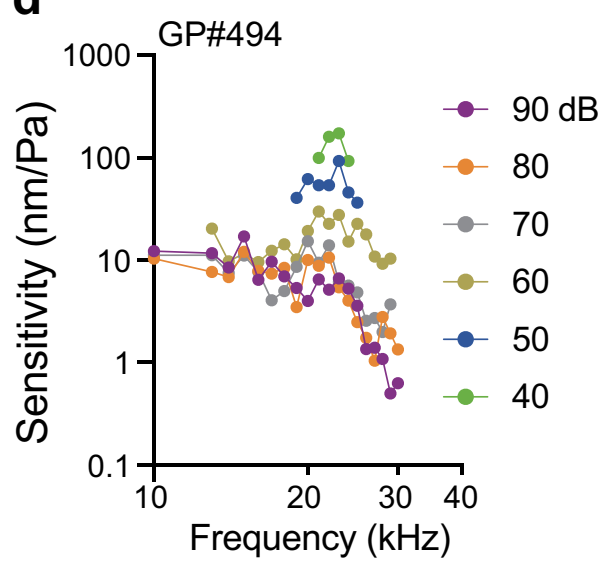

different frequencies (10 and $13-30 \mathrm{kHz}$ spaced by $1 \mathrm{kHz}$ ) and sound pressure (40-90 dB SPL; $10 \mathrm{~dB}$ steps), while the responses of the cochlear partition at the basal turn were monitored by the modified SPM interferometer. In panels $\mathbf{c}$ and $\mathbf{d}$, the vibration amplitude and sensitivity - which was obtained by dividing the amplitude by sound pressure - are plotted against the stimulus frequency, respectively. In this preparation, the best frequency (BF) was $23 \mathrm{kHz}$. See the main text for the definition of the BF and Supplementary Text in Online Resource 3 for the detailed description of the results 
channels as a function of the hair bundle deflection in outer hair cells [30, 32]. Moreover, the nonlinear behaviour of the cochlear partition leads to nonlinear distortion and distortionproduct otoacoustic emissions (DPOAEs), among which the cubic ones are dominant $[40,43]$.

Recently, however, Doppler vibrometry combined with optical coherence tomography (OCT) has demonstrated quadratic distortion in the vibration profile of the region including outer hair cells but not in the vibration profile of the basilar membrane near the cochlear base [35, 49]. A quadratic nonlinearity can create a static offset in response to a pure tone. In support of this notion, the electrophysiological recordings from the basal turn have demonstrated an offset in the sinusoidally oscillating membrane potential of outer hair cells and in the cochlear microphonic potential, which primarily mirrors the current flowing through the mechanosensitive channels $[12,22]$. In addition, in the apical turn, a static offset of 1$200 \mathrm{~nm}$ in the vibration of the cochlear partition has been measured with a laser interferometer [7]. Similar motion has also been detected from Reissner's membrane and from the tectorial membrane overlaying hair cells $[9,42]$. Nevertheless, none of the studies involving OCT vibrometry have yet described slow vibrations at frequencies below $100 \mathrm{~Hz}$ in the partition, possibly owing to a limitation of the methodology or of the algorithm of data analysis [11, 27, 28, 35, 49].

In this study, we therefore aimed to directly measure and investigate the static offset in the vibration of the cochlear partition in the basal turn of live guinea pigs. For this purpose, we modified sinusoidal phase modulating (SPM) laser interferometry, a technique that can accurately detect the target's movement at low frequencies including $0 \mathrm{~Hz}$ at a high signalto-noise ratio [46]. Our in vivo measurements confirmed the presence of a nanoscale offset in the travelling wave. Moreover, we found that the magnitude depended upon the intensity and frequency of the stimulation. Finally, pharmacological experiments implied the involvement of somatic motility by outer hair cells in the offset.

\section{Materials and methods}

\section{The laser interferometer}

The laser interferometry used in this study was developed on the basis of the SPM method [46]. The interferometer was integrated into a commercial microscopy system (BX61WI; Olympus, Tokyo, Japan) as shown in Supplementary Fig. 1a (Online Resource 1). A light source was provided by a nearinfrared laser diode (wavelength $780 \mathrm{~nm}$; linewidth $2 \mathrm{MHz}$; maximum power $15 \mathrm{~mW}$; LP780-SAD15; Thorlabs, NJ, USA). This laser diode contained an optical isolator and optical fibre. The light intensity at the end of the fibre was fixed at $12.6 \mathrm{~mW}$ by a laser controller (CLD1011; Thorlabs, NJ,
USA). A parallel beam of light was emitted from a collimator lens (F810APC-780; Thorlabs, NJ, USA) connected to the fibre. This arrangement reduced the light intensity to $11.1 \mathrm{~mW}$. The parallel light was divided into two beams in beam splitter I (90/10 Beamsplitter; Chroma, VT, USA); one beam was provided for the interferometer $(9.99 \mathrm{~mW})$, and the other was directed towards a CMOS camera (AdvanCam18HR; Olympus, Tokyo, Japan). The former was split by beam splitter II (CCM1-BS014/M; Thorlabs, NJ, USA) equally into a reference beam and object beam, which were used to irradiate a reference mirror (NE530B; Thorlabs, NJ, USA) and a sample object, respectively, through objective lenses (XLFluor 4×/340; Olympus, Tokyo, Japan). Each lens was placed at a distance of $2.8 \mathrm{~cm}$ from the mirror or the object. The light intensity measured at the termination of each beam was $3.3 \mathrm{~mW}$. The beams that were reflected from the mirror and sample were coupled together in beam splitter II and were detected by interference at a photodetector (PDA100A-EC; Thorlabs, NJ, USA) that was placed at a distance of $24 \mathrm{~cm}$ from the beam splitter. The gain and the low-pass cut-off frequency of the detector were set to $40 \mathrm{~dB}(\times 100)$ and $225 \mathrm{kHz}$, respectively. A piezo-actuator (AE0505D18; Thorlabs, NJ, USA) was attached to the reference mirror. In this context, the actuators that we used originated from two different lots, $\mathrm{A}$ and $\mathrm{B}$. To sinusoidally move the mirror, the actuator was stimulated with the AC voltage evoked by means of a function generator (AFG-1022; Tektronix, OR, USA) as follows: for lot $\mathrm{A}$, the frequency was $45 \mathrm{kHz}$ and the peak-to-peak voltage was $11,13,15,17$, or $20 \mathrm{~V}$ (the experiments in Supplementary Figs. 1 and 2 and experiments on the four animals: GP\#235, 257, 258, and 396); for lot B, the frequency was $49 \mathrm{kHz}$ and the peak-to-peak voltage was $20 \mathrm{~V}$ (the other experiments). In any case, the initial phase was set to $60^{\circ}$ or $70^{\circ}$. The induced vibration amplitude was $16.9-25.0 \mathrm{~nm}$. The function generator was driven by a trigger pulse generated by a computer that had a sampling rate of $204.8 \mathrm{kHz}$ (NI PXI-4461; National Instruments, TX, USA). The pulse generation was controlled by a software that we wrote in LabVIEW (LabVIEW 2013 Service Pack 1, 32-bit; National Instruments, TX, USA).

Note that in this study, we modified the SPM method to simultaneously measure the sinusoidal vibrations and offset in the target. The details of this arrangement are described in Online Resource 2.

\section{Performance evaluation experiments}

The accuracy of measurement of the offset with the modified SPM method (see Online Resource 2) was verified with a piezo-actuator attached to a strain gauge (PZS001; Thorlabs, NJ, USA). This actuator expanded in parallel with the direction of the applied DC voltage. An AC voltage of $21 \mathrm{kHz}$, $0.28 \mathrm{~V}$ peak to peak, and a DC voltage of $0,0.025,0.05$, $0.075,0.1$, or $0.25 \mathrm{~V}$ were simultaneously applied to the 
actuator for $18 \mathrm{~ms}$ as electrical stimulation (Supplementary Fig. 1b). The difference in the actuator's length recorded for $5 \mathrm{~ms}$ before and during the stimulation was quantified by a strain gauge method. The resistance in the gauge was converted by an amplifier (AMP002; Thorlabs, NJ, USA) to voltage, which was analysed on a commercial strain gauge reader (KSG101; Thorlabs, NJ, USA). At each voltage, the actuator was stimulated 80 times, and the average of the measurements was taken as a data point. Subsequently, the change of the actuator's length was examined by the modified SPM method too, with the same stimulus protocol as in the strain gauge method and the workflow described in Supplementary Fig. 1e and in Supplementary Text in Online Resource 3. Finally, the values obtained by the two methods were compared (Supplementary Fig. 1f and g).

Additional evaluation for the modified SPM interferometry was carried out as follows. Suppose that the sample is sinusoidally vibrated, and its amplitude is gradually enlarged without the offset. In homodyne vibrometry, when the sample's vibration amplitude is close to $\lambda / 8$, i.e. approximately $100 \mathrm{~nm}$ in our system $(\lambda=780 \mathrm{~nm})$, a nonlinear relation is observed between the change of interference signal intensity and that of the vibration amplitude $[25,29]$. This non-linearity may apparently induce an offset in the measurement. Nonetheless, we confirmed that this artefact was barely detectable by the SPM interferometry when the vibration amplitude of a piezoelectric element ranged from approximately 0.1 to $50 \mathrm{~nm}$ (Supplementary Fig. 2a). Indeed, in this experiment, the observed background offset values fell within a small range of \pm $0.5 \mathrm{~nm}$ (Supplementary Fig. 2b). It is noteworthy that the measured vibration amplitudes of acoustically stimulated cochlear partitions never exceeded $50 \mathrm{~nm}$.

\section{The animal experiments}

In accordance with the ethics guidelines (see Compliance with ethical standards), guinea pigs (see the next subsection) were housed at the animal facility in the Niigata University School of Medicine. The animals were kept on a $12 \mathrm{~h} \mathrm{light} / 12 \mathrm{~h}$ dark cycle and were provided with ad libitum access to food and water. All the experiments were conducted during the light phase. The animal handling and reporting complied with the ARRIVE guidelines [26].

\section{Animal preparation for in vivo experiments}

For in vivo assays, 141 male Hartley guinea pigs were used (200$580 \mathrm{~g} ; 2-8$ weeks old; SLC, Shizuoka, Japan). All the following experiments were carried out in an acoustically and electrically shielded room. Guinea pigs were deeply anesthetised with intraperitoneal injection of urethane $\left(1.5 \mathrm{~g} \mathrm{~kg}^{-1}\right)$. The toe pinch, corneal reflexes, and respiratory rate were examined to evaluate the depth of anaesthesia. When anaesthesia was insufficient, additional urethane was injected into the animals at $0.3 \mathrm{~g} \mathrm{~kg}^{-1}$. During the experiment, the body temperature of each animal was kept at $38^{\circ} \mathrm{C}$ by means of a heating pad (Deltaphase Isothermal Pad; Braintree Scientific, MA, USA). The temperature of the cochlea was additionally controlled by supplemental heat to the head from a lamp [34]. After tracheotomy, which was intended for the maintenance of spontaneous breathing, an anterior portion of the bulla was opened with a ventral approach to expose the basal turn of the cochlea [36]. Then, the tensor tympani muscle was cut with a sharp scalpel [33]. Additionally, a fenestra was made on the lateral site of the bulla in order to shine the laser light onto a spot of the basilar membrane constituting the surface of the cochlear partition through the round window membrane. The pinna was resected. The head of a guinea pig was fixed on an acrylic plate $(50 \times 20 \times$ $5 \mathrm{~mm}$ ) with two M2.5 screws. The plate was tightly connected to the stage (SL20/M; Thorlabs, NJ, USA). In this process, the angle and position of the cochlea were manually controlled to let the laser beam irradiate the cochlear partition through the round window membrane as perpendicularly as possible.

\section{Acoustic stimuli}

To examine the auditory brainstem response (ABR) and motions in the cochlear partition, tone-burst sounds were generated from a speaker (EC1; Tucker-Davis Technologies, FL, USA) with its driver (ED1; Tucker-Davis Technologies, FL, USA). The driver was controlled by the application of AC voltage between $0.1 \mathrm{mV}$ and $10 \mathrm{~V}$. The input port of a Yshaped waveguide was connected to the exit of the speaker. This set-up enabled us to symmetrically divide the propagation route of the sound into two paths. Each output port of the waveguide was attached to an ear tip (ER10D-T03; Etymotic Research Inc., IL, USA). One tip was tightly inserted into the left external ear canal of an animal. The other tip was next connected to a different type of ear tip (ER10D-T013; Etymotic Research Inc., IL, USA), which had been assembled with a microphone (ER-10B+; Etymotic Research Inc., IL, USA). This microphone, which had been calibrated beforehand with a sound calibrator (Type2127; ACO CO, LTD, Tokyo, Japan), was employed to measure the intensity of the sound from the speaker. Protocols of acoustic stimuli for each series of the assays are described in Supplementary Fig. 3a and b, Supplementary Text in Online Resource 3, and below.

\section{Measurement of motions in the cochlear partition}

Before the tensor tympani muscle of each guinea pig was cut and after measurement of the motions in an intact cochlear partition, the ABR was examined as previously described [47] (see Supplementary Table 1). The procedure for ABR measurement is described in Supplementary Methods (Online Resource 2). 
The motions of the cochlear partition were analysed by the modified SPM interferometry as follows. A laser beam was directed through the round window membrane to the cochlear partition 1.5-2.0 mm apically from the basal extreme of each cochlea [29] (Fig. 1a). The target for the beam was determined by the appearance of a shadowed belt, which is likely to correspond to the region of outer hair cells [4] (Fig. 1b). The illuminated area was approximately $900 \mu^{2}$. Although the laser was focused upon the basilar membrane, interference signals were theoretically evoked from not only the membrane but also the other components of the cochlear partition; the signals were gathered in the photodetector of the modified SPM interferometer. The protocols and workflow for measurement of the motions in the cochlear partition are illustrated in Supplementary Figs. $1 \mathrm{e}, 3 \mathrm{a}$, and $\mathrm{b}$ and in the Supplementary Text in Online Resource 3. Of note, before examination of the partition's motion in each cochlea, we focused the microscope on the round window membrane and confirmed that the voltage of the interference signal detected at the same frequency as that of acoustic stimulation did not exceed the limit of detection (LOD) [29].

As presented in Fig. $1 \mathrm{c}$ and d, a tuning curve was constructed for a guinea pig to verify our animal preparation and experimental approach (for details, see Supplementary Text in Online Resource 3). In other in vivo experiments, the offset of the cochlear partition was characterised through the following process. Firstly, for an individual guinea pig, tuning curves were constructed in advance by the stimulus protocol illustrated in Supplementary Fig. 3a (40, 60, and 80 dB SPL; $10 \mathrm{~s}$ in total for stimulation) to identify the best frequency (BF) $[18$, 39] (for the definition of the BF, see the 'Results' section). Secondly, the LOD of the interference signals at the BF was evaluated by means of 80 sets of $5 \mathrm{~ms}$ recordings without stimulation. The third step was the measurement of the offset. The acoustic stimulation consisted of a tone-burst sound with a 1-ms rising phase, 16-ms duration, and a 1-ms falling phase, followed by an $82-\mathrm{ms}$ interval; the 5 -ms data recorded in the absence and presence of the stimulation were analysed to characterise the motions in the cochlear partition (Supplementary Fig. 3b). For the stimulation at the BF, the intensity was varied from 50 to $90 \mathrm{~dB}$ SPL in $10 \mathrm{~dB}$ increments (Fig. 2b and c). Alternatively, for examination of frequency dependence of the offset (Fig. 3b and Supplementary Fig. 4), the sounds at a constant intensity of $90 \mathrm{~dB}$ SPL were applied to an animal while the frequency was changed (15$24 \mathrm{kHz} ; 1 \mathrm{kHz}$ steps). All these offset measurements were carried out by means of basically the same procedure and workflow as those used for the pilot experiment in Supplementary Fig. 1c-e.

Out of the 141 guinea pigs examined in this study, we excluded animal preparations and a series of acquired data from the analyses under any one of the following nine conditions. Firstly, the experiment was terminated when the ABR threshold measured before cutting of the tensor tympani muscle exceeded $30 \mathrm{~dB}$ SPL (25 animals) (for details, see Online Resource 2). The second condition was the case where we found a rupture in the ear drum when dissecting the tensor tympani muscle (four animals). The third issue was low intensity of interference signals from the cochlear partition at the outset of each experiment; this problem was attributed to obstruction of laser irradiation of the target position by an overlay of a styloid process upon the round window membrane (27 animals). Fourthly, we discarded the preparations in which stimulus intensity for acquisition of data for the threshold tuning curve was $\geq 60 \mathrm{~dB}$ SPL (16 animals). In the fifth condition, during the measurements of the cochlear partition, the object's interference signals (Supplementary Fig. 1d) induced by $60 \mathrm{~dB}$ stimuli did not exceed the LOD under control conditions (three animals). In the sixth case, the ABR threshold measured immediately before euthanasia of an animal (Figs. 2 and 3) or administration of salicylate crystals (sodium salicylate; Wako, Osaka, Japan; Fig. 4) exceeded 50 dB SPL (six animals). Seventhly, total experiment time for an animal exceeded $4 \mathrm{~h}$ (one animal), the time limit for keeping a guinea pig's conditions stable [19]. Eighthly, in the tuning curves, the peak position for the amplitude of sinusoidal vibrations did not shift to a lower frequency as the stimuli were intensified (seven animals). Finally, 42 animals died during the experiments. Accordingly, the number of the animals described in the 'Results' section of the main text is 10 .

\section{Statistical analysis}

Means \pm SD served as descriptive statistics. Statistical significance of the data in Fig. 4 was determined with the paired $t$ test (Prism 8; GraphPad Software, San Diego, CA, USA). Data with a $p$ value of $<0.05$ were considered significant. The $p$ values were subjected to Bonferroni's correction for multiple comparisons.

\section{Results}

\section{Detection and characterisation of the offset in the cochlear partition}

We constructed an interferometer by combining two previously described methodologies: the SPM technique, which is intended for the measurement of static offsets [46], and homodyne interferometry, which quantifies sinusoidal vibrations [25] (Supplementary Fig. 1a-e). Although few applications of the original SPM method have been reported, experiments with a piezoelectric element confirmed the accuracy and efficacy of our interferometer for quantitative detection of offsets (Supplementary Fig. 1f and g). Interferometry could determine in which direction a sample's position moved: 
a

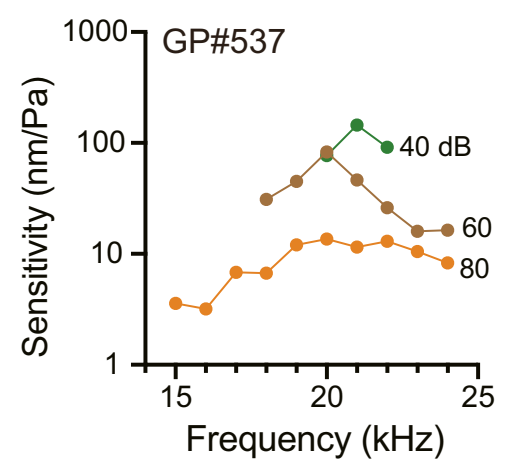

b

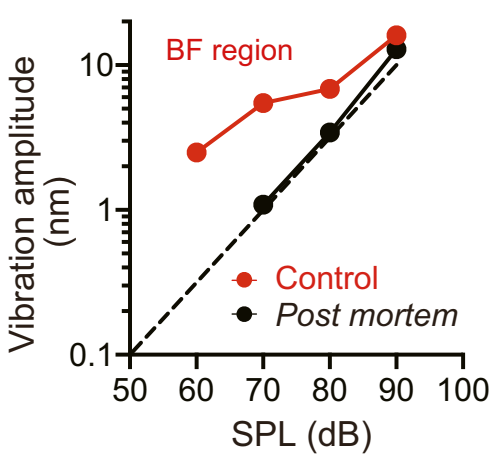

C

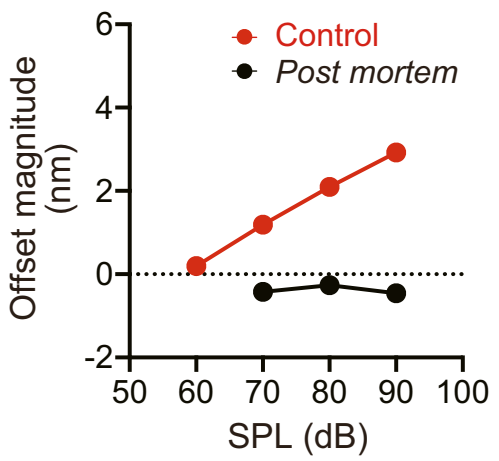

Fig. 2 Measurement of an offset in the cochlear partition. For this guinea pig (GP\#537), the tuning curves were initially obtained with pure toneburst sounds at 40,60, and $80 \mathrm{~dB}$ SPL (a). At each intensity, the frequency was changed from 15 to $24 \mathrm{kHz}$ in $1 \mathrm{kHz}$ steps. The stimulus protocol was the same as that illustrated in Fig. 1c and d (see Supplementary Fig. 3a). In accordance with the threshold tuning curve, the best frequency (BF) for this animal was found to be $21 \mathrm{kHz}$. The vibration amplitude and offset magnitude were then simultaneously recorded in the same animal under control (red) and post mortem (black) conditions; the respective results were plotted in panels $\mathbf{b}$ and $\mathbf{c}$ as a function of the sound pressure level (SPL). In panel $\mathbf{b}$, although the amplitude of sinusoidal vibrations grows linearly post mortem, under

offsets towards the scala vestibuli or the scala tympani were represented by positive or negative values, respectively (see Online Resource 2).

By means of the interferometer we constructed, we sought to examine sound-induced motions in cochlear partitions in the high-frequency region of guinea pig cochleae. The amplitude of the partition's sinusoidal vibrations at the basal turn of the cochlea is much less than that at the apical turn [43]. Because we expected that the offset of the target region would be relatively small and affected by even subtle perturbations, we adopted a minimally invasive experimental approach. A laser beam was directed through the transparent round window membrane at the cochlear partition $1.5-2.0 \mathrm{~mm}$ apically from the basal extreme of an intact cochlea [29] (Fig. 1a and b). In addition, we established criteria for successful measurements as described in the 'Materials and methods' section. The properties of the tuning curve obtained for sinusoidal vibrations with this experimental approach and tone-burst stimuli composed of pure sounds $(10-30 \mathrm{kHz})$ are similar to those described elsewhere [10,51] (Fig. 1c and d).

Then, in a different guinea pig, we intended to stimulate the intact cochlea with pure tone-burst sounds at the BF, which induced a maximal amplitude in the threshold tuning curve constructed in advance [18]. Recording of the tuning curves by the same stimulus protocol as that depicted in Fig. 1 (10$30 \mathrm{kHz}, 10 \mathrm{~dB}$ increments; Supplementary Fig. 3a) took at least $2-3 \mathrm{~h}$; therefore, a series of experiments including animal preparation with the surgical procedure and measurement of the partition's motions under control conditions could not be completed within $4 \mathrm{~h}$, the time limit that ensured an animal's control circumstances, the data fit a power law relation with a slope of approximately $0.3 \mathrm{~dB} / \mathrm{dB}$ indicative of compressive non-linearity. The dashed line in $\mathbf{b}$ indicates linear growth. Note that in panel $\mathbf{c}$, the offset magnitude under control conditions monotonically increases as the stimulus increases; this behaviour clearly differs from the post mortem response that represents the noise level of the measurements. The data depicted in panels $\mathbf{b}$ and $\mathbf{c}$ were acquired according to the workflow described in Supplementary Fig. 1e; a stimulus cycle consisted of a tone-burst sound with a 1-ms rising phase, 16-ms duration, and a 1-ms falling phase, followed by an $82-\mathrm{ms}$ interval (see Supplementary Fig. 3b). The summary flowchart for this series of experiments is displayed in Supplementary Fig. 3c

intact hearing threshold [19]. We therefore modified the protocol for tuning curves as follows. Firstly, the range of the stimulus frequencies was narrowed to a window of 15 $24 \mathrm{kHz}$. Secondly, the sound intensity was increased in $20 \mathrm{~dB}$ steps from $40 \mathrm{~dB}$ SPL. The BF determined by this procedure was $21 \mathrm{kHz}$ under control conditions (Fig. 2a). Next, in the same animal, we succeeded in measuring not only sinusoidal vibrations but also the offset with a different stimulus protocol (Fig. 2b and c; for the protocol, see Supplementary Fig. 3b). The threshold intensity for detection of sinusoidal vibrations was $60 \mathrm{~dB}$ SPL, which exceeded the level in the evaluation experiment (40 dB SPL; see Figs. 1c, d and 2a). This impaired sensitivity was attributed to an elevation of the detection limit by the ultrashort recording period (5 ms; Supplementary Fig. 3b), which minimised the influence of low-frequency noise such as breathing and heartbeats in live animals. The vibration amplitude grew less steeply as sound pressure increased from 60 to $90 \mathrm{~dB}$, manifesting the typical profile of compressive non-linearity (Fig. 2b) [43]. During stimulation at $60 \mathrm{~dB}$ SPL, we simultaneously detected an offset of $0.2 \mathrm{~nm}$ towards the scala vestibuli (Fig. 2c). As the sound intensified, the magnitude was likely to increase monotonically $(1.2 \mathrm{~nm}$ at $70 \mathrm{~dB}$ SPL, $2.1 \mathrm{~nm}$ at $80 \mathrm{~dB}$ SPL, and $2.9 \mathrm{~nm}$ at $90 \mathrm{~dB}$ SPL).

After the animal had been euthanised, the amplitude of the sinusoidal vibrations evoked by stimulation $(21 \mathrm{kHz})$ grew linearly with sound pressure (Fig. 2b) [5, 6]. In addition, the offset decreased markedly for stimuli of 70-90 dB SPL; the magnitude was $-0.4 \mathrm{~nm}$ at $70 \mathrm{~dB},-0.3 \mathrm{~nm}$ at $80 \mathrm{~dB}$, and $0.5 \mathrm{~nm}$ at $90 \mathrm{~dB}$ (Fig. 2c). In this situation, $60 \mathrm{~dB}$ stimuli did 


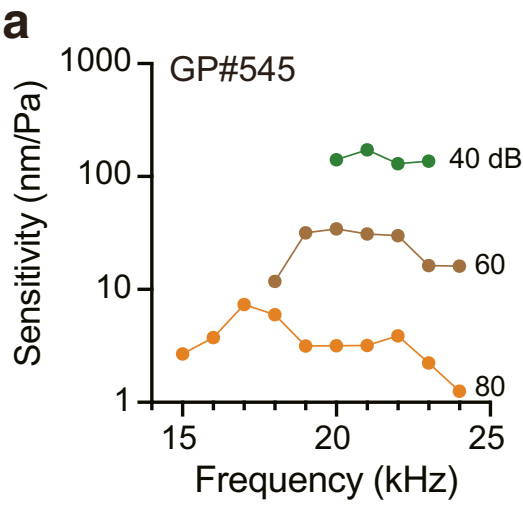

Fig. 3 Frequency dependence of the offset. In this series of experiments, a guinea pig (GP\#545), whose BF was found to be $21 \mathrm{kHz}$ by means of the tuning curves obtained in advance (a), was exposed to $90 \mathrm{~dB}$ acoustic stimuli at different frequencies $(15-24 \mathrm{kHz}$ in $1 \mathrm{kHz}$ increments), and motions of the cochlear partition were measured by the SPM interferometry. In panel $\mathbf{b}$, the offset magnitudes are plotted as a function of stimulus frequencies. Under control conditions, the offset was prominent at the stimulus frequencies that elicited strong nonlinearity in panel a $(21-24 \mathrm{kHz})$; at other frequencies, the responses

not induce a motion exceeding the threshold of detection. These observations indicate that the offset is dependent upon cellular activity sensitive to a loss of metabolic energy.

We further examined the effects of changing stimulus frequency upon the offset. A guinea pig, whose $\mathrm{BF}$ was found in advance to be $21 \mathrm{kHz}$ (Fig. 3a), was exposed to $90 \mathrm{~dB}$ puretone sounds at different frequencies $(15-24 \mathrm{kHz}$; for the stimulus protocol, see Supplementary Fig. 3b). The offset peaked at $23 \mathrm{kHz}$ and decayed on both sides of the peak (Fig. 3b). Clear responses that exceeded $1.0 \mathrm{~nm}$ were observed at $\geq$ $21 \mathrm{kHz}$ (Fig. 3b). In the sinusoidal vibrations within this range, we observed prominent non-linearity as well as sensitivity exceeding $100 \mathrm{~nm} \mathrm{~Pa}^{-1}$ at $40 \mathrm{~dB}$ SPL (see Fig. 3a). On the other hand, the offsets detected at $\leq 18 \mathrm{kHz}$ were subtle: 0.2 to $0.3 \mathrm{~nm}$. These values approximately fell into the range that was determined post mortem in the same animal (from 0.3 to $0.3 \mathrm{~nm}$ ). Similar results were observed in two other animals (Supplementary Fig. 4). Accordingly, it seems probable that the offset is evoked at frequencies near the BF, and the magnitude depends upon stimulation frequency.

\section{The mechanism underlying the offset of the cochlear partition}

We attempted to identify the factor that critically contributes to the offset detected in the motion of the cochlear partition. Previous measurements by OCT vibrometry indicated that the quadratic distortion product in response to tone-complex stimuli at multiple frequencies occurs in a region including outer hair cells $[35,49]$. In addition to this observation, the disappearance of the offset post mortem (Figs. 2c and 3b) prompted us to examine the involvement of the active process

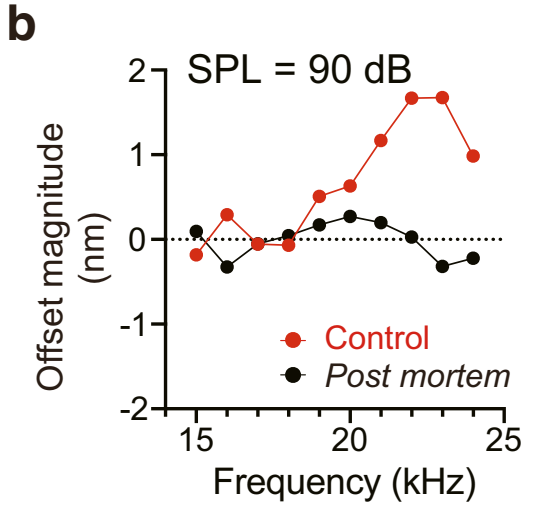

diminished to the level similar to that of the post mortem measurements, which represents the noise floor. For the tuning curves (a), acoustic stimuli of 40,60 , or $80 \mathrm{~dB}$ SPL at varying frequencies (15-24 kHz in $1 \mathrm{kHz}$ steps) were tested with the protocol illustrated in Supplementary Fig. 3a. The data depicted in panel $\mathbf{b}$ were acquired according to the workflow described in Supplementary Fig. 1e; a stimulus cycle consisted of a tone-burst sound with a 1-ms rising phase, 16-ms duration, and a 1-ms falling phase, followed by an 82-ms interval (see Supplementary Fig. 3b)

of outer hair cells, which is responsible for the compressive non-linearity observed in sinusoidal vibrations of a healthy basilar membrane [33]. Because somatic motility in hair cells plays a key role in the active process and depends on the motor protein prestin [52], we pharmacologically blocked this protein's activity and compared the partition's motions with those under control and post mortem conditions (Fig. 4).

In this series of assays as well, it was necessary to complete all the recordings in individual live animals within $4 \mathrm{~h}$ [19]. To shorten the time of the experiment, we therefore omitted the determination of tuning curves and used $21 \mathrm{kHz}$ pure toneburst sounds for stimulation. This frequency was expected to be close to the $\mathrm{BF}$ in the target region for the laser beam, according to the tuning curves in the foregoing four guinea pigs (Figs. 2 and 3 and Supplementary Fig. 4). Under control conditions, we again clearly observed not only the compressive non-linearity of the vibration amplitude, a typical phenomenon detected with stimuli at the BF, but also a significant offset in five animals (Fig. 4a and b; Supplementary Fig. 5). Note that the offset magnitude increased monotonically with sound pressure; this trend was similar to that obtained in Fig. 2c with stimuli at the measured BF $(21 \mathrm{kHz})$. Crystals of salicylate, a blocker of prestin, were subsequently placed onto the round window membranes in the individual cochleae [31, 41]. Twenty minutes later, the motions of the partitions were again examined. The amplitude of the sinusoidal vibrations depended linearly upon the strength of stimulation [31] $(n=$ 5; Fig. 4a). In addition, the offset towards the scala vestibuli was prominently attenuated at 80 and $90 \mathrm{~dB}$ SPL (Fig. 4b; control versus salicylate administration for the offset; $p=$ 0.0054 and $t=7.396$ at $80 \mathrm{~dB}, p=0.0312$ and $t=4.555$ at $90 \mathrm{~dB}$; according to the paired $t$ test with Bonferroni's 


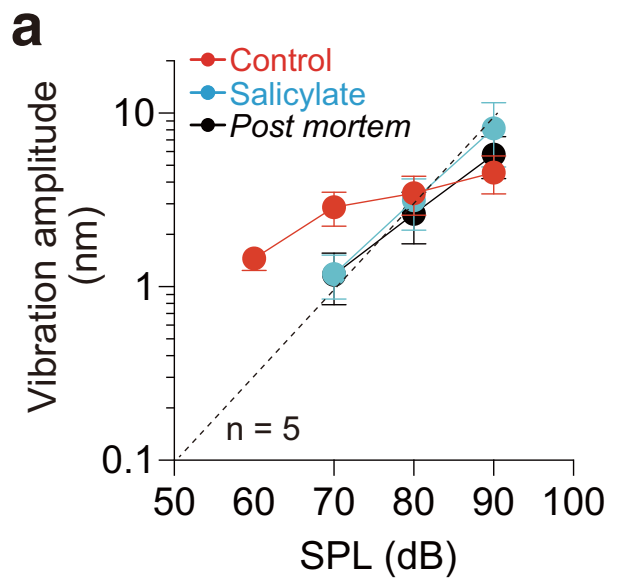

Fig. 4 Effects of pharmacological perturbation of somatic motility in outer hair cells. The sound-evoked motions of the cochlear partitions were measured by means of the SPM interferometer in five guinea pigs under control (red), salicylate administration (blue), and post mortem (black) conditions as indicated in the insets. The stimulus frequency was constant at $21 \mathrm{kHz}$. The procedure for the pharmacological perturbation is described in the main text. In panels $\mathbf{a}$ and $\mathbf{b}$, dots and error bars (mean $\pm \mathrm{SD}$ ) denote the amplitude of sinusoidal vibrations (a) and the magnitude of the offset (b), which are plotted as a function of sound pressure levels (SPLs; $n=5$ ). In panel a, the vibration amplitude obtained under control conditions is characterised by compressive nonlinearity; therefore, $21 \mathrm{kHz}$ is likely to preserve the effects of the $\mathrm{BF}$ in these animal preparations (see Fig. 2b). The amplitude measured post mortem and during salicylate treatment shows a passive response and

correction for multiple comparisons). The values of the two parameters at 70-90 dB SPL with the pharmacological perturbation were comparable with the level characteristic of post mortem conditions (Fig. 4a and b). The offset is therefore likely to depend on the somatic motility of outer hair cells.

\section{Discussion}

Cooper and Rhode [8] placed reflective microbeads on the basilar membrane at the basal turn of the guinea pig cochlea and examined the travelling wave by means of an interferometer. When stimulating this cochlear portion with pure-tone sound at a high frequency, they detected a negligible static offset, which can be defined as $0 \mathrm{~Hz}$ motion. Recent studies using OCT systems indicate that at the basal turn, the quadratic distortion is produced by stimuli in the region comprising outer hair cells [35, 49], implying the presence of the offset in these cells. As for multi-tone sounds used in such works, different frequency components should induce individual offsets, and these responses must be mixed. To quantitatively assess the phenomenon of a static offset, we chose in this study stimulation at a single frequency. The SPM interferometry system that we utilised has a prominent property: the algorithm designed for the measurements and data analyses

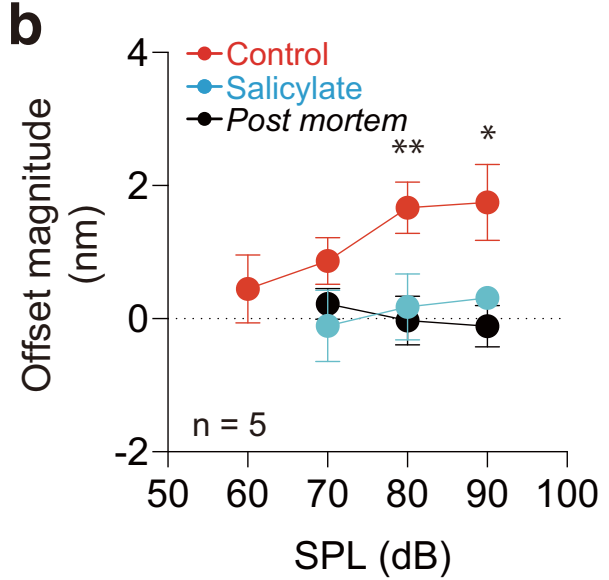

loses the property of compressive non-linearity. The dashed line in panel $\mathbf{a}$ indicates linear growth. In panel $\mathbf{b}$, the individual values of the offset detected with stimuli of different intensities under the three conditions were plotted and statistically compared by the paired $t$ test with Bonferroni's correction for multiple comparisons $(* p<0.05$, $* * p<0.01$; control versus salicylate treatment $p=0.1659, t=2.679$ for $70 \mathrm{~dB}, p=0.0054, t=7.396$ for $80 \mathrm{~dB}, p=0.0312, t=4.555$ for $90 \mathrm{~dB}$; salicylate treatment versus post mortem conditions $p=0.8637, t=1.225$ for $70 \mathrm{~dB}, p=0.8094, t=1.280$ for $80 \mathrm{~dB}, p=0.0999, t=3.188$ for $90 \mathrm{~dB}$ ). This series of data (b) points to a prominent reduction in the offsets by euthanasia or by the application of salicylate. All the individual measurements used for the analyses of $\mathbf{a}$ and $\mathbf{b}$ are displayed in Supplementary Fig. 5

allowed us to extract slow motions of $0-100 \mathrm{~Hz}$, which have not yet been addressed by OCT vibrometers. Our system automatically discarded significant background movements induced by heartbeats, respiration, and muscle contractions from the measurements in real time to improve the signal-tonoise ratio of the data (see Supplementary Methods in Online Resource 2). Due to this advantage, our interferometer was suitable for the purpose of this study, although the methodology used here could not determine which cell type(s) within the cochlear partition were involved.

Although the offset that we detected in the cochlear basal turn was only a few nanometres, it is likely to be significant for several reasons. In each animal, we could not determine the range of background fluctuations of the intact cochlear partition in a resting state because the recording period of the experimental protocol including this evaluation inevitably exceeded $4 \mathrm{~h}$, which is the limit that guarantees an intact hearing threshold of these animals [19]. Nevertheless, in the 18 measurements under control conditions with stimuli of 70 $90 \mathrm{~dB}$ SPL in the six guinea pigs analysed in Figs. 2 and 4, 15 data points for the offset exceeded $1 \mathrm{~nm}$. In all 15 cases, the response was clearly impaired by salicylate treatment of the cochleae or by euthanasia; the magnitudes then fell into a range of -0.8 to $+0.9 \mathrm{~nm}$. Values of approximately $\pm 1 \mathrm{~nm}$ were likely to represent the maximal background noise and 
thus the threshold for accurate detection in our in vivo preparations. Accordingly, in the experiment presented in Fig. 3b, the offset measurements at $\geq 21 \mathrm{kHz}$ - the range that included the $\mathrm{BF}$ - was significant. Moreover, during the pharmacological treatment and post mortem (Figs. 2c and $4 \mathrm{~b}$ and Supplementary Fig. 5), the data at $60 \mathrm{~dB}$ SPL could not be plotted. The motion was likely to have been suppressed by each of the two perturbations to a level too low to be detected.

In the cochlear basal turn, the amplitude of the quadratic distortion product with stimuli of $94 \mathrm{~dB}$ SPL exceeded that for stimuli of $74 \mathrm{~dB}$ SPL [35]. In support of this finding, we observed that the partition's offset grew monotonically with sound pressure (Figs. 2c and 4b; Supplementary Fig. 5). Moreover, the response peaked at a stimulus frequency near the BF (Fig. 3b and Supplementary Fig. 4). Similar intensity and frequency dependences of the offset are also observed for Hensen's cells in the cochlear apical turn of guinea pigs [7]. Therefore, this pattern may be conserved throughout the cochlea. The maximal offset that we measured in the high-pitch region with stimuli of $90 \mathrm{~dB}$ SPL was approximately $3 \mathrm{~nm}$ for the $\mathrm{BF}$ of $21 \mathrm{kHz}$ (Fig. 2c), which is much smaller than the value of $130 \mathrm{~nm}$ in the low-pitch region for BFs of 0.6$0.9 \mathrm{kHz}$ [7]. This difference could be due to a gradient of the morphological and physical properties of the basilar membrane and the overlying cellular components along the cochlea [13]. Note that at the target portion of the apical turn, the offset is peaked at the stimuli of $\sim 0.2 \mathrm{kHz}$, which is separated from the BF by $70-80 \%$ of this frequency [7]. On the other hand, we found that the gap was $\sim 10 \%$ in the basal turn (Fig. $3 \mathrm{~b}$ and Supplementary Fig. 4). The elements underlying this discrepancy remain uncertain and thereby would be analysed by further work.

Although the quadratic distortion product in the partition's vibrations induced by multi-tone stimuli is likely to emerge from the activity of outer hair cells [35, 49], the underlying mechanism has not yet been identified. We therefore examined the effect on the partition's motion of salicylate, which is commonly used as an inhibitor of somatic motility of outer hair cells $[1,22,23,38,48]$. The results with this reagent (Fig. 4) indicate that in response to acoustic stimulation, a steady contraction mediated by prestin occurs in the hair cell soma, and this action likely underlies the offset of the cochlear partition. Nevertheless, the pharmacological targets should be addressed carefully because of the following observations. In outer hair cells, somatic motility depends upon membrane potential [3, 23]. This electrical property is controlled by cation influx through mechanosensitive channels localised at the tip of the hair bundle [24]. Because application of salicylate reduces the amplitude of the channels' current to some extent in vitro [24], this compound might affect active hair bundle motility. Moreover, administration of the compound attenuates blood flow in the vessels serving the lateral cochlear wall, which is connected with the cochlear partition [16]. This effect might change physical properties of the lateral wall and thus contribute to the elimination of the offset by the drug. Further experiments are needed to clarify how much factor(s) other than the somatic motility of hair cells are involved in offset induction.

The measurements in Fig. 4 implied that a steady contraction of the outer hair cells occurs in vivo and contributes to the offset of the cochlear partition. How this cellular motion is induced remains elusive. One possibility is that acoustic stimuli yield an offset of hair bundles. Alternatively, somatic motility might be regulated by organisation of the activity of efferent neurons innervating the outer hair cells. In any case, the steady change of hair cells' length should control cellular mechanical activity, and this modification should be fed back to the offset of the cochlear partition.

An observation in a classical electrophysiological experiment suggests a possible physiological role for the offset. The amplitude of the cochlear microphonic, which primarily represents the current flowing through outer hair cells [17], temporarily diminishes when positive hydrostatic pressure is applied to the perilymph in the scala tympani, a perturbation that likely shifts the cochlear partition towards the scala vestibuli [53]. The hair cell's current excites the cells and thereby elicits somatic motility [2]. Our interferometric measurement showed that the partition's offset towards the scala vestibuli was well detectable with stimuli of 80 and $90 \mathrm{~dB}$ SPL (Figs. $2 \mathrm{c}$ and $4 \mathrm{~b}$; Supplementary Fig. 5). The offset might accordingly affect the hair cell's somatic motility and attenuate the amplification of the sinusoidal vibrations of the cochlear partition when an animal is exposed to loud sounds. This idea points to a contribution of the offset to enhancement of the nonlinear compression of the vibration amplitude and thus to the broad dynamic range associated with normal hearing. The second possible significance of the offset is its relation to the machinery that prevents damage to fine cochlear structures by loud sounds. A similar role is proposed for the acoustic reflex of the stapedial muscle in the middle ear, but this action is negligible at stimulus frequencies exceeding $5 \mathrm{kHz}$ [44]. The offset in the cochlear partition could serve as a fundamental system of protection from acoustic trauma. These kinds of possible functional significance of the partition's offset should be verified in future studies.

Acknowledgements We thank Dr. A.J. Hudspeth (The Rockefeller University) and Dr. Elizabeth S. Olson (Columbia University) for their valuable comments on the experiments and their critical reading of the manuscript.

Author contributions $\mathrm{HH}, \mathrm{FN}, \mathrm{SK}$, and KatD conceived of the study. TO, SS, GO, MPS, and TT designed the experiments. TO, FN, and SC constructed the interferometer. TO, SM, KenD, SK, and TR analysed the experimental data. TO and $\mathrm{HH}$ wrote the manuscript. All the authors reviewed and approved the final manuscript. 
Funding information This work is supported by the following research grants and funds: AMED-CREST 19gm0810004h, AMED (to HH, SC, and SK); Grant-in-Aid for Scientific Research A 18H04062 (to HH), Grantin-Aid for Scientific Research B 18H02950 (to FN), Grant-in-Aid for Scientific Research B 19H02151 (to SC), Grant-in-Aid for Scientific Research S 18H05242 (to SK), and Grant-in-Aid for Young Scientists B 17K17736 (to SS) from the Ministry of Education, Culture, Sports, Science and Technology of Japan; Yamaguchi Educational and Scholarship Foundation J18G0050 (to TO); the Collaborative Research Program of Institute for Protein Research, Osaka University, CR-19-03 (to HH); and by the Joint Research Program of the Biosignal Research Center, Kobe University (281003) (to HH).

\section{Compliance with ethical standards}

Conflict of interest The authors declare that they have no conflict of interest.

Ethics approval (research involving animals) All the protocols for animal experiments in this study were approved by the President of Niigata University (Permission Number: \#28 Niigata Univ. Res. 64-5). In vivo assays were carried out in compliance with the protocol reviewed by the Institutional Animal Care and Use Committee and with the Japanese Animal Protection and Management Law.

Informed consent Not applicable.

Open Access This article is licensed under a Creative Commons Attribution 4.0 International License, which permits use, sharing, adaptation, distribution and reproduction in any medium or format, as long as you give appropriate credit to the original author(s) and the source, provide a link to the Creative Commons licence, and indicate if changes were made. The images or other third party material in this article are included in the article's Creative Commons licence, unless indicated otherwise in a credit line to the material. If material is not included in the article's Creative Commons licence and your intended use is not permitted by statutory regulation or exceeds the permitted use, you will need to obtain permission directly from the copyright holder. To view a copy of this licence, visit http://creativecommons.org/licenses/by/4.0/.

\section{References}

1. Ashmore J (2008) Cochlear outer hair cell motility. Physiol Rev 88: 173-210. https://doi.org/10.1152/physrev.00044.2006

2. Ashmore J, Avan P, Brownell WE, Dallos P, Dierkes K, Fettiplace R, Grosh K, Hackney CM, Hudspeth AJ, Julicher F, Lindner B, Martin P, Meaud J, Petit C, Santos-Sacchi J, Sacchi JR, Canlon B (2010) The remarkable cochlear amplifier. Hear Res 266:1-17. https://doi.org/10.1016/j.heares.2010.05.001

3. Ashmore JF (1987) A fast motile response in guinea-pig outer hair cells: the cellular basis of the cochlear amplifier. J Physiol 388:323347. https://doi.org/10.1113/jphysiol.1987.sp016617

4. Brundin L, Flock A, Khanna SM, Ulfendahl M (1991) Frequencyspecific position shift in the guinea pig organ of Corti. Neurosci Lett 128:77-80. https://doi.org/10.1016/0304-3940(91)90763-j

5. Chen F, Zha D, Fridberger A, Zheng J, Choudhury N, Jacques SL, Wang RK, Shi X, Nuttall AL (2011) A differentially amplified motion in the ear for near-threshold sound detection. Nat Neurosci 14:770-774. https://doi.org/10.1038/nn.2827
6. Cooper NP (1998) Harmonic distortion on the basilar membrane in the basal turn of the guinea-pig cochlea. J Physiol 509(Pt 1):277288. https://doi.org/10.1111/j.1469-7793.1998.277bo.x

7. Cooper NP, Dong W (2003) Baseline position shifts and mechanical compression in the apical turns of the cochlea. Paper presented at the Biophysics of the Cochlea, pp 261-270

8. Cooper NP, Rhode WS (1992) Basilar membrane mechanics in the hook region of cat and guinea-pig cochleae: sharp tuning and nonlinearity in the absence of baseline position shifts. Hear Res 63: 163-190 PMID: 1464568

9. Cooper NP, Rhode WS (1995) Nonlinear mechanics at the apex of the guinea-pig cochlea. Hear Res 82:225-243 PMID: 7775288

10. Cooper NP, Rhode WS (1997) Mechanical responses to two-tone distortion products in the apical and basal turns of the mammalian cochlea. J Neurophysiol 78:261-270. https://doi.org/10.1152/jn. 1997.78.1.261

11. Cooper NP, Vavakou A, van der Heijden M (2018) Vibration hotspots reveal longitudinal funneling of sound-evoked motion in the mammalian cochlea. Nat Commun 9:3054. https://doi.org/10. 1038/s41467-018-05483-Z

12. Corns LF, Johnson SL, Kros CJ, Marcotti W (2014) Calcium entry into stereocilia drives adaptation of the mechanoelectrical transducer current of mammalian cochlear hair cells. Proc Natl Acad Sci U S A 111:14918-14923. https://doi.org/10.1073/pnas.1409920111

13. Dallos P (1992) The active cochlea. J Neurosci 12:4575-4585 PMID: 1464757

14. Dallos $P$ (2008) Cochlear amplification, outer hair cells and prestin. Curr Opin Neurobiol 18:370-376. https://doi.org/10.1016/j.conb. 2008.08.016

15. Dallos P, Popper AN, Fay RR (1996) The cochlea. Springer Handbook of Auditory Research, vol 8. Springer, New York

16. Didier A, Miller JM, Nuttall AL (1993) The vascular component of sodium salicylate ototoxicity in the guinea pig. Hear Res 69:199 206. https://doi.org/10.1016/0378-5955(93)90108-d

17. Fettiplace R (2017) Hair cell transduction, tuning, and synaptic transmission in the mammalian cochlea. Compr Physiol 7:11971227. https://doi.org/10.1002/cphy.c160049

18. He W, Kemp D, Ren T (2018) Timing of the reticular lamina and basilar membrane vibration in living gerbil cochleae. Elife 7: e37625. https://doi.org/10.7554/eLife.37625

19. He W, Ren T (2013) Basilar membrane vibration is not involved in the reverse propagation of otoacoustic emissions. Sci Rep 3:1874. https://doi.org/10.1038/srep01874

20. Hibino H, Kurachi Y (2006) Molecular and physiological bases of the $\mathrm{K}^{+}$circulation in the mammalian inner ear. Physiology (Bethesda) 21:336-345. https://doi.org/10.1152/physiol.00023. 2006

21. Hudspeth AJ (2014) Integrating the active process of hair cells with cochlear function. Nat Rev Neurosci 15:600-614. https://doi.org/ $10.1038 / \mathrm{nrn} 3786$

22. Jacob S, Pienkowski M, Fridberger A (2011) The endocochlear potential alters cochlear micromechanics. Biophys J 100:25862594. https://doi.org/10.1016/j.bpj.2011.05.002

23. Kakehata S, Santos-Sacchi J (1996) Effects of salicylate and lanthanides on outer hair cell motility and associated gating charge. J Neurosci 16:4881-4889 PMID: 8756420

24. Kennedy HJ, Evans MG, Crawford AC, Fettiplace R (2006) Depolarization of cochlear outer hair cells evokes active hair bundle motion by two mechanisms. J Neurosci 26:2757-2766. https://doi. org/10.1523/JNEUROSCI.3808-05.2006

25. Khanna SM (1986) Homodyne interferometer for basilarmembrane measurements. Hear Res 23:9-26. https://doi.org/10. 1016/0378-5955(86)90172-3

26. Kilkenny C, Browne WJ, Cuthill IC, Emerson M, Altman DG (2010) Improving bioscience research reporting: the ARRIVE 
guidelines for reporting animal research. PLoS Biol 8:e1000412. https://doi.org/10.1371/journal.pbio.1000412

27. Kim W, Kim S, Huang S, Oghalai JS, Applegate BE (2019) Picometer scale vibrometry in the human middle ear using a surgical microscope based optical coherence tomography and vibrometry system. Biomed Opt Express 10:4395-4410. https:// doi.org/10.1364/BOE.10.004395

28. Lee HY, Raphael PD, Park J, Ellerbee AK, Applegate BE, Oghalai JS (2015) Noninvasive in vivo imaging reveals differences between tectorial membrane and basilar membrane traveling waves in the mouse cochlea. Proc Natl Acad Sci U S A 112:3128-3133. https:// doi.org/10.1073/pnas.1500038112

29. Lukashkin AN, Bashtanov ME, Russell IJ (2005) A self-mixing laser-diode interferometer for measuring basilar membrane vibrations without opening the cochlea. J Neurosci Methods 148:122129. https://doi.org/10.1016/j.jneumeth.2005.04.014

30. Mellado Lagarde MM, Drexl M, Lukashkina VA, Lukashkin AN, Russell IJ (2008) Outer hair cell somatic, not hair bundle, motility is the basis of the cochlear amplifier. Nat Neurosci 11:746-748. https://doi.org/10.1038/nn.2129

31. Murugasu S, Russell IJ (1995) Salicylate ototoxicity: the effects on basilar membrane displacement, cochlear microphonics, and neural responses in the basal turn of the guinea pig cochlea. Audit Neurosci 1:139-150

32. Nin F, Reichenbach T, Fisher JA, Hudspeth AJ (2012) Contribution of active hair-bundle motility to nonlinear amplification in the mammalian cochlea. Proc Natl Acad Sci U S A 109:2107621080. https://doi.org/10.1073/pnas.1219379110

33. Nuttall AL, Dolan DF, Avinash G (1991) Laser Doppler velocimetry of basilar membrane vibration. Hear Res 51:203-213 PMID: 1827786

34. Nuttall AL, Guo M, Ren T (1999) The radial pattern of basilar membrane motion evoked by electric stimulation of the cochlea. Hear Res 131:39-46. https://doi.org/10.1016/s0378-5955(99) 00009-x

35. Nuttall AL, Ricci AJ, Burwood G, Harte JM, Stenfelt S, CayeThomasen P, Ren T, Ramamoorthy S, Zhang Y, Wilson T, Lunner T, Moore BCJ, Fridberger A (2018) A mechanoelectrical mechanism for detection of sound envelopes in the hearing organ. Nat Commun 9:4175. https://doi.org/10.1038/s41467-018-06725-w

36. Ogata G, Ishii Y, Asai K, Sano Y, Nin F, Yoshida T, Higuchi T, Sawamura S, Ota T, Hori K, Maeda K, Komune S, Doi K, Takai M, Findlay I, Kusuhara H, Einaga Y, Hibino H (2017) A microsensing system for the in vivo real-time detection of local drug kinetics. Nat Biomed Eng 1:654-666. https://doi.org/10.1038/s41551-017-01185

37. Oghalai JS (2004) The cochlear amplifier: augmentation of the traveling wave within the inner ear. Curr Opin Otolaryngol Head Neck Surg 12:431-438. https://doi.org/10.1097/01.moo. 0000134449.05454 .82

38. Oliver D, He DZ, Klocker N, Ludwig J, Schulte U, Waldegger S, Ruppersberg JP, Dallos P, Fakler B (2001) Intracellular anions as the voltage sensor of prestin, the outer hair cell motor protein. Science 292:2340-2343. https://doi.org/10.1126/science.1060939
39. Olson ES (1998) Observing middle and inner ear mechanics with novel intracochlear pressure sensors. J Acoust Soc Am 103:34453463. https://doi.org/10.1121/1.423083

40. Reichenbach T, Hudspeth AJ (2014) The physics of hearing: fluid mechanics and the active process of the inner ear. Rep Prog Phys 77:076601. https://doi.org/10.1088/0034-4885/77/7/076601

41. Ren TY, He WX, Barr-Gillespie PG (2016) Reverse transduction measured in the living cochlea by low-coherence heterodyne interferometry. Nat Commun 7:10282. https://doi.org/10.1038/ ncomms 10282

42. Rhode WS, Cooper NP (1996) Nonlinear mechanics in the apical turn of the chinchilla cochlea in vivo. Audit Neurosci 3:101-121

43. Robles L, Ruggero MA (2001) Mechanics of the mammalian cochlea. Physiol Rev 81:1305-1352. https://doi.org/10.1152/physrev. 2001.81.3.1305

44. Rosowski JJ, Ravicz ME, Songer JE (2006) Structures that contribute to middle-ear admittance in chinchilla. J Comp Physiol A 192: 1287-1311. https://doi.org/10.1007/s00359-006-0159-9

45. Ruggero MA, Rich NC (1991) Application of a commerciallymanufactured Doppler-shift laser velocimeter to the measurement of basilar-membrane vibration. Hear Res 51:215-230. https://doi. org/10.1016/0378-5955(91)90038-b

46. Sasaki O, Okazaki H (1986) Sinusoidal phase modulating interferometry for surface profile measurement. Appl Opt 25:3137-3140. https://doi.org/10.1364/Ao.25.003137

47. Sato MP, Higuchi T, Nin F, Ogata G, Sawamura S, Yoshida T, Ota T, Hori K, Komune S, Uetsuka S, Choi S, Masuda M, Watabe T, Kanzaki S, Ogawa K, Inohara H, Sakamoto S, Takebayashi H, Doi K, Tanaka KF, Hibino H (2017) Hearing loss controlled by optogenetic stimulation of nonexcitable nonglial cells in the cochlea of the inner ear. Front Mol Neurosci 10:300. https://doi.org/10. 3389/fnmol.2017.00300

48. Tunstall MJ, Gale JE, Ashmore JF (1995) Action of salicylate on membrane capacitance of outer hair cells from the guinea-pig cochlea. J Physiol 485(Pt 3):739-752. https://doi.org/10.1113/ jphysiol.1995.sp020765

49. Vavakou A, Cooper NP, van der Heijden M (2019) The frequency limit of outer hair cell motility measured in vivo. Elife 8:e47667. https://doi.org/10.7554/eLife.47667

50. Von Békésy G (1960) Experiments in hearing. McGraw-Hill series in psychology. McGraw-Hill, New York,

51. Zha D, Chen F, Ramamoorthy S, Fridberger A, Choudhury N, Jacques SL, Wang RK, Nuttall AL (2012) In vivo outer hair cell length changes expose the active process in the cochlea. PLoS One 7:e32757. https://doi.org/10.1371/journal.pone.0032757

52. Zheng J, Shen W, He DZ, Long KB, Madison LD, Dallos P (2000) Prestin is the motor protein of cochlear outer hair cells. Nature 405: 149-155. https://doi.org/10.1038/35012009

53. Zou Y, Zheng J, Ren T, Nuttall A (2006) Cochlear transducer operating point adaptation. J Acoust Soc Am 119:2232-2241. https:// doi.org/10.1121/1.2173517

Publisher's note Springer Nature remains neutral with regard to jurisdictional claims in published maps and institutional affiliations. 